\title{
A disciplina de educação física promovendo saúde na escola: um exame bibliográfico
}

\author{
The discipline of physical education promoting health at school: a bibliographic \\ examination
}

\section{La disciplina de la educación física que promueve la salud en la escuela: un examen bibliográfico}

José Roberto Gonçalves de Abreu ${ }^{1}$, Cláudia Mariano Simões ${ }^{1 *}$, Renan Rocha Camilato Knop ${ }^{1}$, Jobério Nunes Coutinho Junior ${ }^{1}$, Kadjas Púperi Monteiro ${ }^{1}$, Elida Gangá Viana Alves ${ }^{1}$, Fernanda Aparecida da Silva1 ${ }^{1}$ Luana Evangelista Laurent' ${ }^{1}$, Vinicius da Silva Freitas ${ }^{1}$, Cíntia Pancieri.

\section{RESUMO}

Objetivo: É objetivo deste exame revisional, apresentar os pontos fortes já estudados acerca de como a disciplina de educação física promove saúde no ambiente de ensino. Revisão Bibliográfica: A disciplina de educação física já não pode ser considerada apenas o momento da diversão e da descontração como por muito tempo se fez. Por mais que exista o quebrar da formalidade da sala de aula e o deslocamento do ambiente comum de aplicação das disciplinas, há que se considerar todos os aspectos importantes que levam a valorização da prática de esportes. Manter o corpo em movimento é, na maioria das vezes, manter o corpo saudável. A prática de esportes em um País que a maior parte da população é sedentária, surge como alternativa no o combate a doenças causadas pela estagnação do corpo, sobrepeso, entre outras consequências de não manter um ritmo mínimo de atividades físicas. Considerações Finais: O estudo é pautado na divulgação e na proposição de ideias que levem a esclarecer a importância da prática de esportes do ambiente escolar, principalmente no que se refere aos seus benefícios para a saúde.

Palavras-chave: Saúde e educação, Saúde na escola, Esporte e saúde.

\begin{abstract}
Objective: The objective of this review is to present the strengths already studied about how the discipline of physical education promotes health in the teaching environment. Bibliographic Review: The physical education discipline can no longer be considered just the moment of fun and relaxation as it has been for a long time. As much as there is a break in the formality of the classroom and a shift in the common environment in which disciplines are applied, it is necessary to consider all the important aspects that lead to the valorization of the practice of sports. Keeping the body in motion is, for the most part, keeping the body healthy. The practice of sports in a country where most of the population is sedentary, appears as an alternative in combating diseases caused by stagnation of the body, overweight, among other consequences of not maintaining a minimum pace of physical activities. Final Considerations: The study is based on the dissemination and proposition of ideas that lead to clarifying the importance of practicing sports in the school environment, especially with regard to its health benefits.
\end{abstract}

Key words: Health and education, School health, Sports and health.

\section{RESUMEN}

Objetivo: El objetivo de esta revisión es presentar las fortalezas ya estudiadas sobre cómo la disciplina de la educación física promueve la salud en el ambiente de enseñanza. Revisión Bibliográfica: La disciplina de educación física ya no puede considerarse solo el momento de diversión y relajación como lo ha sido durante mucho tiempo. Por mucho que haya una ruptura en la formalidad del aula y un desplazamiento del entorno común para la aplicación de disciplinas, es necesario considerar todos los aspectos importantes que conducen a la valorización de la práctica deportiva. Mantener el cuerpo en movimiento es, en su mayor parte, mantener el cuerpo sano. La práctica del deporte en un país donde la mayoría de la población es sedentaria, aparece como una alternativa para combatir enfermedades causadas por el estancamiento del cuerpo, el sobrepeso, entre otras consecuencias de no mantener un ritmo mínimo de actividades físicas. Consideraciones Finales: El estudio se basa en la difusión y propuesta de ideas que conducen a aclarar la importancia de practicar deportes en el entorno escolar, especialmente en lo que respecta a sus beneficios para la salud.

Palabras clave: Salud y educación, Salud escolar, Deportes y salud.

${ }^{1}$ Faculdade Vale do Cricaré (FVC), São Mateus - ES. *E-mail: claudiasimoes.adv@gmail.com 


\section{INTRODUÇÃO}

A falta de atividade física nas últimas décadas tem contribuído para o aumento do sedentarismo e agravos à saúde e ao bem-estar do indivíduo, todos decorrentes de um novo padrão de vida na sociedade moderna. A literatura descreve a relação entre exercício físico e saúde de crianças e adolescentes.

Os dois aspectos que promovem a participação de jovens em atividades esportivas são esportes e participação em aulas de educação física. Em geral, ambos estão relacionados à saúde dos jovens. O exercício físico é inversamente proporcional ao excesso de peso, e a participação na educação física é diretamente proporcional à saúde cardiorrespiratória e ao comportamento saudável (COLEDAM DHC, et al., 2014).

$\mathrm{Na}$ atenção básica à saúde, foram institucionalizados o conteúdo e as ações de educação física, o que indica mudanças nos métodos de trabalho em saúde e maior convergência com esse campo. Nesse sentido, a aproximação do conceito de "saúde ampliada" pode ajudar a orientar a aparência dos profissionais em um campo específico, permitindo que eles se envolvam em trabalho multidisciplinar com outros profissionais (FREITAS FF, et al., 2014).

Os dados acerca da obesidade precoce e como ela assola às comunidades internacionais foram divulgados pela Organização Mundial da Saúde (OMS), e apontam que em 1974 a prevalência de excesso de peso em adolescentes era de $4 \%$, e passou para $30 \%$ no ano de 2010 . Os dados corroboram com a redução do número de crianças e adolescentes que praticam esportes ou atividades físicas, já que a inserção tecnológica atraiu esse público para um estilo de vida bem mais sedentário.

Como a obesidade pode agir como agravante de outras doenças e até mesmo ser fatal, é necessário se propor soluções, e a educação física é uma forma de mostrar ao público infanto-juvenil alternativas para uma vida saudável (CUREAU FV, et al., 2012). Geralmente, quando o tópico em discussão é educação física, o pensamento popular passa a ser sinônimo de momento desperdiçado ou descanso para os alunos, poucos conseguem observar de maneira aberta uma educação física para promover a saúde e a qualidade de vida.

Dentro do plano de aula, se atendo a realidade, esportes competitivos e de alto desempenho são ensinados como parte dos esportes escolares na sala de aula, quase sempre conduzidos por pesquisas intensificadas para atingir metas que refletem necessariamente a educação e os interesses e necessidades gerais de aprendizagem dos alunos, além de expor os efeitos benéficos a saúde corporal, que é entendido atualmente como sinônimo de prática de esportes. Na educação física escolar, essa deve ser a maior intenção e preocupação (SANTOS J e OLIVEIRA EL, 2015).

Historicamente, a atividade física sempre esteve em um nível mais baixo em comparação com a atividade intelectual reconhecida. Nesse sentido, dois aspectos levaram à desvalorização da educação física. $O$ primeiro refere-se a uma maneira simplificada de visualizar a área, que está no campo da atividade constante e não de observar e identificar conhecimentos específicos. O segundo refere-se ao senso comum presente no pensamento familiar sobre as pessoas e seus hábitos físicos, dando a falsa impressão de que, mesmo no ambiente escolar, a área e os profissionais são quase que dispensáveis (JUNIOR NB e TASSONI ECM, 2013).

O exercício físico é um expressivo indicador da saúde do adolescente. Está relacionado à maior aptidão cardiorrespiratória, força muscular, redução de gordura corporal e melhor metabolismo cardiovascular e estado mental. Recomenda-se fornecer diferentes tipos de exercícios físicos para crianças e jovens, um dos quais é participar de aulas de educação física. Trata-se de um curso de ensino fundamental e médio, cujos objetivos incluem promover a saúde através do exercício físico durante as aulas e manter os alunos ativos durante toda a vida. Estudos têm demonstrado que o esporte contribui para a saúde dos estudantes de diferentes maneiras. As aulas aumentam a atividade física diária geral, bem como exercícios moderados a vigorosos (COLEDAM DHC, et al., 2017).

Intenciona-se nesta revisão apresentar os pontos fortes da disciplina de educação física no ambiente escolar como instrumentos de promoção de uma vida saudável e mais regrada no que concerne à saúde do aluno. É importante também, relatar as políticas públicas que já existem no sentido de transformar a educação 
física em uma disciplina mais instrutiva no que tange a práticas de esportes e apontar novos caminhos já descritos na literatura, à fim de induzir analiticamente às pessoas aos verdadeiros conceitos dessa disciplina, impedindo a propagação dos conceitos comuns infundados, de que se trata de disciplina dispensável ou pouco instrutiva.

\section{REVISÃO BIBLIOGRÁFICA}

Considerando que ações e práticas que priorizam a população envolvem aspectos como sistemas de saúde, educação, trabalho e desenvolvimento social, promover a saúde e o bem-estar de crianças e adolescentes é um grande desafio. A contribuição do esporte e da escola é a base desse processo, sendo possível a educação baseada na promoção da saúde, para que os alunos possam reconhecer e estimular a importância de o exercício físico regular como principal forma de prevenção de doenças e garantir um melhor equilíbrio corporal (SANTANA DP e COSTA CRB, 2016).

Ainda para Santana DP e Costa CRB (2016), as práticas atuais trouxeram inúmeros benefícios à sociedade, mas levaram a profundas transformações sociais e econômicas. De acordo com as necessidades e características especiais de cada época, os indivíduos que costumavam permanecer ativos hoje se tornam inertes, adaptáveis e sedentários. A redução no exercício físico e nos hábitos de lazer associados ao uso constante de computadores, telefones celulares, dispositivos eletrônicos e automóveis (incluindo mudanças na dieta, baixa qualidade nutricional e alimentos ricos em gordura) tem tentado crianças e jovens a mudar de corpo e diretamente intervir na qualidade de vida. Todas essas práticas mudaram os hábitos da população, que apresenta um alto índice de doenças e obesidade.

Acredita-se que, embora a educação física seja considerada parte do currículo no campo da linguagem e esteja naturalmente sujeita a ideias educacionais que controlam o escopo da escola, especialistas da área acreditam que uma das tarefas da educação física deve ser a criação de meios que sejam benéficos para os alunos na promoção da sua saúde como, conhecimento corporal, desenvolvimento de hábitos benéficos ao organismo humano, alimentação saudável e outros temas relacionados à saúde e a qualidade de vida. Aceitando que o ambiente escolar é um espaço para se propagar conhecimentos úteis aos seres humanos, a ideia é induzir o pensamento de que é muito importante discutir a relação entre saúde e esporte nas escolas (GUIMARÃES CCPA, et al., 2015).

Com as mudanças na epidemiologia, nutrição e demografia, é necessário mudar a estratégia de atenção à saúde da população brasileira. Portanto, as pessoas percebem a importância de alguns profissionais envolvidos na expansão dos processos de saúde. Entre eles, os profissionais de educação física apresentam o ideal de promover a saúde e contatar a escola como componente de aprendizado do currículo (OLIZ MM, et al., 2020).

No tocante as políticas públicas de esporte e lazer, a busca é para que a educação física se torne, assim como nos países da América do Norte, um incentivador de jovens a buscar o esporte. O início das carreiras esportivas depende do incentivo e da oportunidade, ainda se tratando de um país subdesenvolvido, o Brasil necessita do aprimoramento das suas estruturas para desenvolver através da disciplina de educação física, uma ampliação massiva da prática de esportes.

Fator que colaboraria para a ampliação desse possível projeto, seria o ensino em tempo integral, que abriria espaço para um maior contato do aluno com a prática esportiva, além de ampliar as possibilidades de planejamento docente (STAREPRAVO FA, et al., 2013).

A legislação brasileira, dispõe na sua Carta Magna de 1988, dispositivos que indicam o direito fundamental ao esporte e ao lazer. Infelizmente a realidade é outra, mas a determinação do legislador original não é imperativa ou taxativa a ponto de não se permitir ser cumprida por desleixo dos gestores pretéritos do Estado que não cumpriram com suas obrigações necessárias.

É possível dar início a novos processos de políticas sociais à fim garantir no plano físico os direitos resguardados pela Constituição Federal (CF). É precioso enfatizar que muitas legislações versam acerca dos direitos da criança e do adolescente no que tange ao esporte, cultura e lazer, todos esses direitos consagrados primordialmente pela CF (LOPES ACB e BERCLAZ MS, 2019). 
A Constituição determina o seguinte: Artigo 217. É dever do Estado fomentar práticas desportivas formais e não-formais, como direito de cada um, observados: I a autonomia das entidades desportivas dirigentes e associações, quanto a sua organização e funcionamento; II - a destinação de recursos públicos para a promoção prioritária do desporto educacional e, em casos específicos, para a do desporto de alto rendimento; III - o tratamento diferenciado para o desporto profissional e o nãoprofissional; IV - a proteção e o incentivo às manifestações desportivas de criação nacional (BRASIL, 1988).

Outro aspecto importante é que os professores podem ajudar a moldar papéis e influenciar na aquisição de comportamentos e padrões de estilo de vida, disseminando assim o que acreditam. Isso nos leva a questioná-los sobre sua compreensão sobre saúde física. O consenso entre os professores é que eles estão de acordo com o que preconiza a Organização Mundial da Saúde, manter a harmonia, manter a prática regular de exercícios físicos, o lazer e os hábitos saudáveis.

Este conceito tem sido amplamente difundido na mídia e aceito pelas pessoas, mas o desafio é transformálo em ações específicas no ambiente escolar e transformá-lo no interesse dos alunos, para que possa ser efetivamente integrado à vida adulta por meio das seguintes modalidades: o desenvolvimento de habilidades pessoais e sociais necessárias para escolher comportamentos (AZAMBUJA CR, et al., 2017).

\section{Educação física e a promoção da saúde na escola}

Para Alencar GMP, et al. (2017), durante muito tempo, o termo saúde foi considerado apenas sinônimo de livre de doenças, mas com o passar do tempo, a definição se tornou mais ampla e ganhou novo significado, que pode ser definido como bem-estar físico, mental e social. Esses três itens devem estar relacionados. $O$ movimento humano trata-se de um fenômeno que contém sua especificidade e está essencialmente entrelaçado com vários aspectos do corpo humano e sua dimensão ampla.

Desde muito cedo, a prática de esportes e as atividades físicas são vistas sob um ponto de vista que justifica o papel da educação física na grade educacional atualmente. Desde os tempos antigos, os esportes estão ativamente envolvidos no currículo para melhor enfrentar os desafios da vida e da saúde. As pessoas prepararam uma visão melhor da sociedade, da qualidade da vida política, do campo militar e do controle dos comportamentos sociais, visando à manutenção do poder desde então (JUNIOR NB e TASSONI ECM, 2013).

Dúvidas acerca da qualidade e condição de vida, no que se refere à saúde e educação, pode se tornar um desafio no encontro entre as duas áreas. Não importa onde isso aconteça, seja uma escola ou uma instituição médica, a interação entre os dois é uma maneira importante de melhorar a qualidade de vida.

Diante das necessidades da escola, a construção de práticas de ensino relacionadas a essa interação é um enorme desafio. A prática de atividades físicas incentivadas pela disciplina de educação física e o incentivo ao esporte nas disciplinas que versam sobre saúde, são considerados laços importantíssimos das duas áreas (CARVALHO IFFB, 2015).

O esporte e as atividades esportivas foram potencializados no século XIX, após as mudanças políticas e sociais iniciadas no século passado e, desde então, mostram tendência a se tornar uma tela de projeção para a dinâmica social. O exercício físico é uma forma de lazer e recuperação, que pode evitar os efeitos nocivos do trabalho estressante e dos hábitos do cotidiano. Após superar o estágio inicial, o exercício geralmente é uma atividade agradável e traz muitos benefícios aos praticantes, desde a melhora do status lipídico do sangue até a autoestima. Uma vida saudável concentra-se na capacidade de sobreviver livre de doenças ou superar as dificuldades de um estado ou condição de morbidade (SILVA RS, et al., 2010).

O esporte e lazer devem ser desenvolvidos como um dos conceitos da educação física nas escolas da educação básica. No entanto, também aumentou o número de escolas brasileiras, que também oferecem atividades esportivas, como atividades extracurriculares durante o dia, chamadas de atividades esportivas escolares, esportes escolares ou aulas de treinamento.

A educação física escolar possui características próprias, com diferentes formas e objetivos; no entanto, se o plano político de ensino da escola for utilizado como meio, eles poderão constituir práticas 
complementares de ensino. Geralmente, acredita-se que, na educação física escolar, os alunos devem entender a diversidade da cultura corporal ou do conteúdo esportivo e considerar o esporte nesses conteúdos (LUGUETTI CN, et al., 2015).

Para Ferreira HS, et al. (2013), o conceito inerente de desempenho profissional afetará seu processo de treinamento. Ao comentar o treinamento de professores de educação física, ele alertou que, sob a orientação do quadro epistemológico da biomecânica moderna, disciplinas como fisiologia e anatomia permeiam a relação com o campo biomédico e com o campo da saúde e herdam a relação com condicionamento e treinamento físico.

A curiosidade dos alunos sobre seus corpos e a descoberta de possibilidades físicas são propícias à aceitação das informações de saúde por eles. Por isso, é de enorme importância fomentar e estruturar a prática e o desenvolvimento da educação física como forma de esclarecer e disseminar as práticas esportivas à fim da busca por uma vida mais saudável.

A Pesquisa Nacional de Saúde Escolar preencheu algumas lacunas na saúde pública do Brasil, especialmente no que diz respeito a atividade e/ou exercício físico. Primeiro, os dados transversais coletados em 2009 representam o primeiro estudo nacional sobre a prática de exercício físico para jovens. Além disso, esses dados podem ser usados como referência para monitorar os hábitos de atividade física dos jovens brasileiros. Nas capitas e no Distrito Federal 60.973 jovens brasileiros foram entrevistados. Quase $90 \%$ dos jovens estudam na 9ª série e têm entre 13 e 15 anos (HALLAL PC, et al., 2010).

A proporção de jovens ativos é de $43,1 \%$ e os meninos $(56,2 \%)$ são maiores que as meninas $(31,3 \%)$. Apenas metade dos adolescentes $(49,2 \%)$ relatou ter recebido duas ou mais aulas de educação física na semana anterior à entrevista, enquanto $79,2 \%$ dos adolescentes relataram assistir TV por mais de duas horas por dia.

Os dados apresentados estão longe de serem satisfatórios, já que menos da metade dos jovens são ativos fisicamente e as futuras gerações, que já são tratadas como propensas ao sedentarismo por não conseguirem equilibrar a atividade corporal com a utilização das novas facilidades tecnológicas, ficam a mercê de fracas políticas de incentivo e estruturação, tanto dos espaços escolares para a prática de esportes, como os centros públicos destinados a prática de esporte e lazer (HALLAL PC, et al., 2010).

\section{Políticas públicas de esporte e lazer como instrumento de potencialização da educação física}

O Ministério do Esporte fez progressos no campo das políticas públicas, criando o Programa Esporte e Lazer da Cidade (PELC) e propondo o treinamento como uma ferramenta educacional para tornar as políticas locais elegíveis aos direitos sociais e atender às necessidades das comunidades relevantes. Entretanto, não é comum a prática de esportes se eles não são conhecidos. É de suma importância incentivar através da possibilidade de prática com instrução, evitando a prática indevida ou de maneira errada, os esportes, investindo ainda em diversidade para que ambos os grupos sociais sejam contemplados (SILVA DS, et al., 2014).

Nos últimos 50 anos, o Brasil está passando por um intenso processo de urbanização. Grande parte $(35,4 \%)$ da população total do Brasil vive em 15 metrópoles, cobrindo 204 das 5.564 cidades do país. Devido a essa transformação acelerada e à influência da revolução tecnológica baseada na tecnologia da informação/comunicação, na formação da economia global e no processo de intercâmbio cultural, é possível perceber a expansão das questões sociais, culturais, econômicas e ambientais (MORETTI AC, et al., 2009).

Portanto, no atendimento prestado pelo departamento de saúde pública ao público, existem inconsistências no atendimento, pois a demanda por doenças crônicas é grande e o mesmo ocorre com o modelo de atendimento às doenças agudas. Nesse universo, revela-se a importância de implementar práticas voltadas à promoção da saúde, ou seja, entender a saúde como um processo e não como ausência da doença, priorizando uma vida de qualidade, defendendo a postura política envolvente e relações sociais mais justas. Dessa forma, a implementação de políticas de esporte e lazer, incentivando o abandono do sedentarismo e induzindo a maiores cuidados com o próprio corpo, torna-se uma grande política de prevenção a doenças crônicas, que em grande parte das vezes surgem através da falta de movimentação do corpo (MORETTI, et al., 2009). 
Nas escolas primárias, a educação física pode ser desenvolvida como um dos conteúdos das práticas esportivas nas escolas. No entanto, o número de escolas que promovem a prática de esportes durante a entressafra está aumentando, o que é chamado de atividades esportivas escolares, ou seja, atividades extracurriculares, também conhecidas como aulas de treinamento esportivo.

Além de fortalecer a educação de crianças e jovens, se eles estiverem efetivamente vinculados às escolas por meio de projetos de educação política, o programa Práticas Esportivas Escolares (PEEs) pode promover a democratização do exercício físico para crianças maiores de seis anos (no caso em que o escolar frequenta a escola). A recomendação parte do artigo $6^{\circ}$ da Lei oㅜ 9.394/96, que determina Diretrizes e Bases da Educação Nacional (LUGUETTI CN, et al., 2011).

A lei 9.393/96 dispõe que: Art. 26. Os currículos da educação infantil, do ensino fundamental e do ensino médio devem ter base nacional comum, a ser complementada, em cada sistema de ensino e em cada estabelecimento escolar, por uma parte diversificada, exigida pelas características regionais e locais da sociedade, da cultura, da economia e dos educandos.

$\S 3^{\circ} \mathrm{A}$ educação física, integrada à proposta pedagógica da escola, é componente curricular obrigatório da educação básica, sendo sua prática facultativa ao aluno: I que cumpra jornada de trabalho igual ou superior a seis horas; II - maior de trinta anos de idade; III - que estiver prestando serviço militar inicial ou que, em situação similar, estiver obrigado à prática da educação física; VI - que tenha prole... (BRASIL, 1996).

No entanto, por outro lado, nos últimos anos, o tema do esporte foi reexaminado de diferentes ângulos e, como o conteúdo do esporte escolar, tende a novas formas de pensar e experimentar. Entre essas possibilidades, não se pode evitar a renovação da tensão esportiva e enfatizar a perspectiva de usar esse tipo de conteúdo para expandir o método de ensino. Naquele momento, as pessoas não apenas buscavam regras técnicas de postura ou reprodução perfeitas, mas também reformavam e reformavam o problema (RICHTER AC, et al., 2011).

A questão da visão social, ainda não se modificou devido aos conhecimentos já relatados que não são inseridos cotidianamente na sociedade. Por muito tempo, a prática de esportes foi apenas reservada a classes sociais mais altas, exceto algumas exceções que obtinham destaque avantajado. $E$ isso fez com que a visão social acerca da educação física e das carreiras esportivas, fossem taxadas como desnecessárias, incentivos ruins ou perda de tempo (COSTA JB, et al., 2016).

Acontece, que devido a essas visões arcaicas, a prática esportiva como necessária ao desenvolvimento humano e importante para a prevenção e contenção de doenças, foi completamente desprezada. Entretanto, é visível que este pensamento está em mutação, assim como muitas outras visões que se desfazem com o avanço do progresso sobre o conservadorismo.

A disciplina de educação física, que por muito foi desvalorizada e tratada como descanso ou momento de diversão apenas, está sendo incorporada ao patamar de indispensável, na visão social, e mantendo o status de lazer, cultura e promoção da saúde pública. Agindo, então, como uma política pública de saúde e desenvolvimento social (COSTA JB, et al., 2016).

\section{CONSIDERAÇÕES FINAIS}

Conclui-se, portanto, que o papel desempenhado pela disciplina de educação física, vai muito além do que ainda prega o conhecimento e a opinião popular. Os benefícios do incentivo à prática de esportes nas escolas, trabalha para reduzir a quantidade exaustiva de alunos sedentários, elevando as condições de saúde dos atores sociais que irão compor a sociedade no futuro. Este estudo contribui para a área do conhecimento, demonstrando que a junção de saúde e educação acontece quando ambas possuem capacidade de solucionar em conjunto problemas sociais. Dentre as inúmeras políticas públicas possíveis e existentes que podem ser desenvolvidas através da disciplina alvo desse estudo, a principal delas é sua existência e valorização, agindo como prática de lazer, saúde, cultura, emprego e renda. 


\section{REFERÊNCIAS}

1. ALENCAR GMP, et al. O desafio da intersetorialidade: processo de implantação do programa saúde na escola no município de Fortaleza - Ceará. Publicado no livro: Atividade física e promoção da saúde na escola: coletânea de estudos, 2017; 7(1): 125-142.

2. AZAMBUJA CR, et al. Percepções de professores de educação física sobre a educação em saúde na escola. Revista Kinesis, 2017; 35(1): 21-27.

3. BRASIL. 1988. Constituição da República Federativa do Brasil: promulgada em 5 de outubro de 1988. 4. ed. São Paulo: Saraiva, 1990.

4. BRASIL. In: LEI 9.394/96, estabelece as diretrizes e bases da educação nacional. 1996.

5. CARVALHO IFFB. A saúde vai à escola: a promoção dasaúde em práticas pedagógicas. Revista de Saúde Coletiva, 2015; 25(4): 1207-1227.

6. COLEDAM DHC, et al. Prática esportiva e participação nas aulas de educação física: fatores associados em estudantes de Londrina, Paraná, Brasil. Cad. Saúde Pública, 2014; 30(3): 533-545.

7. COLEDAM DHC, et al. Aulas de educação física e desfechos relacionados à saúde em estudantes brasileiros. Rev Paul Pediatr., 2017; 36(2): 192-198.

8. COSTA JB, et al. Atividade de ensino na educação física e a formação do pensamento teórico. Psicologia da Educação, 2016; 42(3): 71-80.

9. CUREAU FV, et al. Sobrepeso/obesidade em adolescentes de Santa Maria-RS: prevalência e fatores associados. Revista Brasileira de Cineantropometria \& Desempenho Humano, 2012; 14(5): 517-526.

10. FERREIRA HS, et al. Análise da percepção dos professores de Educação Física acerca da interface entre a saúde e a Educação Física escolar: conceitos e metodologias. Revista Brasileira de Ciências do Esporte, 2013; 35(3): 673685.

11. FREITAS FF, et al. Educação física e saúde: aproximações com a "clínica ampliada". Rev. Bras. Ciênc. Esporte, 2014; 3593): 639-656.

12. GUIMARÃES CCPA, et al. Reflexões sobre Saúde e Educação Física Escolar: a visão dos professores. Revista Hipótese, 2015; 1(4): 113-138.

13. HALLAL PC, et al. Prática de atividade física em adolescentes brasileiros. Ciência \& Saúde Coletiva, 2010; 15(2): 3035-3042.

14. JUNIOR NB, TASSONI ECM. A Educação Física, o docente e a escola: concepções e práticas pedagógicas. Rev Bras Educ Fís Esporte, 2013; 27(3): 467-483.

15. LOPES ACB, BERCLAZ MS. A invisibilidade do Esporte e da Cultura como Direitos da Criança e do Adolescente. Revista Direito e Práxis, 2019; 10(2): 1430-1460.

16. LUGUETTI CN, et al. Gestão de práticas esportivas escolares no ensino fundamental no município de Santos. Revista Brasileira de Educação Física e Esporte, 2011; 25(2): 237-249.

17. LUGUETTI CN, et al. O planejamento das práticas esportivas escolares no ensino fundamental na cidade de Santos. Revista Brasileira de Ciências do Esporte, 2015; 37(4): 314-322.

18. MORETTI AC, et al. Práticas Corporais/Atividade Física e Políticas Públicas de Promoção da Saúde. Saúde Soc, 2009; 18(2): 346-354.

19. OLIZ MM, et al. Utilização de serviços de educação física por adultos e idosos no extremo sul do Brasil: estudo de base populacional, 2020; 25(2): 541-552.

20. PEREIRA MD, OLIVEIRA ESA. Análise do desenvolvimento motor de crianças do $5^{\circ}$ ano do ensino fundamental. Publicado no livro: Atividade física e promoção da saúde na escola: coletânea de estudos, $2017 ; 1$ (1): 16-35.

21. RICHTER AC, et al. Considerações sobre a presença do esporte na educação física infantil: reflexões e experiências. Educar em Revista, 2011; 41(2): 181-195.

22. SANTANA DP, COSTA CRB. Educação física escolar na promoção da saúde. Revista Científica Multidisciplinar Núcleo Do Conhecimento, 2016; 1(10): 171-185.

23. SANTOS J, OLIVEIRA EL. As contribuições do esporte para a educação física escolar. Revista Educação Física UNIFAFIBE, 2015; 4(3): 39-53.

24. SILVA DS, et al. Políticas públicas de esporte e lazer: o processo de formação do Programa Esporte e Lazer da Cidade de Vitória - ES. Revista Brasileira de Ciências do Esporte, 2014; 36(3): 640-647.

25. SILVA RS, et al. Atividade física e qualidade de vida. Ciência \& Saúde Coletiva, 2010; 15(1): 115-120.

26. STAREPRAVO FA, et al. Políticas públicas de esporte e lazer no Brasil: uma argumentação inicial sobre a importância da utilização da teoria dos campos de Pierre Bourdieu. Rev. Bras. Ciênc. Esporte, 2013; 35(3): 785-798. 